\title{
Electrostatically Guided Rydberg Positronium
}

\author{
A. Deller, A. M. Alonso, B. S. Cooper, S. D. Hogan, and D. B. Cassidy \\ Department of Physics and Astronomy, University College London, Gower Street, London WClE 6BT, United Kingdom
}

(Received 10 June 2016; published 12 August 2016)

\begin{abstract}
We report experiments in which positronium (Ps) atoms were guided using inhomogeneous electric fields. Ps atoms in Rydberg-Stark states with principal quantum number $n=10$ and electric dipole moments up to $610 \mathrm{D}$ were prepared via two-color two-photon optical excitation in the presence of a $670 \mathrm{~V} \mathrm{~cm}^{-1}$ electric field. The Ps atoms were created at the entrance of a $0.4 \mathrm{~m}$ long electrostatic quadrupole guide, and were detected at the end of the guide via annihilation gamma radiation. When the lasers were tuned to excite low-field-seeking Stark states, a fivefold increase in the number of atoms reaching the end of the guide was observed, whereas no signal was detected when high-field-seeking states were produced. The data are consistent with the calculated geometrical guide acceptance.
\end{abstract}

DOI: 10.1103/PhysRevLett.117.073202

The ability to manipulate neutral atoms and molecules using optical, electric, or magnetic fields [1-6] has enabled many advances in atomic and molecular physics, such as the creation of new states of matter [7], precision tests of fundamental symmetries [8], ultraprecise atomic clocks [9], cold collision experiments [10], and atom interferometry [11]. Unfortunately positronium (Ps), the hydrogenic electron-positron bound state [12], has not been included in this revolution, largely because of limitations in available positron sources [13] and the short Ps ground-state lifetime: being composed of a particle-antiparticle pair, Ps is inherently unstable in the ground state, with a mean lifetime against annihilation of only 142 ns for the "long-lived" triplet states [14].

This situation is now changing, owing to developments in positron trapping and manipulation techniques [15] that have made high-intensity, pulsed, positron sources considerably more accessible [16]. This in turn facilitates the optical excitation of Ps atoms with pulsed lasers [17], and it is now possible to routinely produce Ps in highly excited (Rydberg) states [18-22]. Since Ps annihilation requires overlap of the electron and positron wave functions, it is strongly suppressed for states with orbital angular momentum quantum number $\ell>0[23,24]$, and the lifetimes of Ps Rydberg-Stark states are almost entirely determined by fluorescence [22]. Thus, transferring Ps atoms from the ground state to Rydberg-Stark states has two beneficial effects: (1) it significantly increases their lifetimes and (2) it can result in the production of atoms with very large electric dipole moments [25]. Together these properties provide a way to control Ps motion using inhomogeneous electric fields [26,27] over long time scales. Variations of such techniques have been used for decades [28] to manipulate ground-state polar molecules [2] as well as atoms and molecules in Rydberg states [4], but have never before been applied to Ps.

Controlling the motion of Ps atoms is desirable because, although there are many physical processes through which
Ps can be generated following positron interactions with solid-state materials [29-34], they all tend to result in similar Ps properties, that is, thermal (or hotter) Ps atoms, emitted with a large angular spread. Because of their low mass $\left(2 m_{e}\right)$ thermal Ps atoms at $300 \mathrm{~K}$ have speeds of $\sim 7 \times 10^{4} \mathrm{~ms}^{-1}$, which is orders of magnitude faster than one can obtain from, for example, a supersonic gas expansion (e.g., [35]) or a buffer gas source (e.g., [36]). Some techniques have been developed to produce colder Ps atoms [37-39], but they are not efficient and/or require unstable targets. Alternative methods under investigation include using cryogenic mesoporous silica films with larger pores $[40,41]$, or Ps production via delocalized Bloch states in metal-organic-framework materials [20]. Currently, however, no satisfactory method is known to efficiently produce Ps atoms with energies $\lesssim 10 \mathrm{meV}$.

The possibility of laser cooling Ps has been discussed for many years [42] but it has not been achieved experimentally. However, when starting from a thermal Ps source, laser cooling would be of only limited utility because viable cooling schemes require transitions that cycle via the ground state (i.e., $1^{3} S \rightarrow 2^{3} P$ transitions), and hence only a finite number of cooling cycles are possible before Ps atoms are lost to annihilation. This amounts to around 30 cycles in $200 \mathrm{~ns}$, and reducing the Ps temperature by $\sim 100 \mathrm{~K}$ would result in the loss of approximately half of the atoms [43]. The laser sources that have been suggested for Ps cooling (e.g., [44]) are generally within current technological capabilities, and laser cooling may become useful if colder ensembles of atoms can be generated, or if other cooling processes can be simultaneously employed (e.g., [45]).

Here we demonstrate radial confinement of Ps atoms in Rydberg-Stark states with principal quantum number $n=10$ in an electrostatic quadrupole guide. The Ps atoms were created in an electric field to facilitate selective excitation of specific parts of the Stark manifold [21]. 
After entering the guide, atoms in states with positive Stark shifts [i.e., low-field-seeking (lfs) Stark states] are deflected away from the electrodes by the inhomogeneous electric fields [46], and are thus guided along the length of the device. Atoms in states with negative Stark shifts [i.e., high-field-seeking (hfs) Stark states] are deflected away from the axis of the guide and are lost. The experiments we report open the door to the future implementation of more sophisticated techniques, including deceleration [47,48] and trapping [49] of Ps atoms.

The experimental methods used to produce and detect Rydberg Ps atoms are the same as those previously described [50]. A Surko-type buffer gas trap [15] is used to produce time-focused [51] positron pulses containing approximately $10^{5}$ particles at a repetition rate of $1 \mathrm{~Hz}$. The temporal and spatial beam properties used here are $\Delta t \sim 4 \mathrm{~ns}$ and $\Delta x \sim 3 \mathrm{~mm}$ (FWHM). The positron beam is transported by a uniform magnetic field of $\sim 100 \mathrm{G}$ and implanted into a mesoporous silica film [32]. This target subsequently emits Ps atoms with a mean longitudinal energy of $\sim 100 \mathrm{meV}$ [52], corresponding to a speed of $\sim 10^{5} \mathrm{~ms}^{-1}$. The Ps production efficiency of the silica targets used is $\sim 25 \%$ [53], and the emitted atoms form a dilute ground-state gas in vacuum with an initial density of approximately $10^{7} \mathrm{~cm}^{-3}$.

Rydberg Ps atoms were prepared using the $1^{3} S \rightarrow 2^{3} P \rightarrow$ $n^{3} S / n^{3} D$ excitation scheme, first demonstrated by Ziock et al. [54]. These transitions were driven by two Nd:YAG pumped pulsed dye lasers (6 ns FWHM): a broadband $\left(\Delta \nu=85 \mathrm{GHz}\right.$,) ultraviolet (UV) laser $\left(E_{\text {pulse }} \approx 500 \mu \mathrm{J}\right.$, $\lambda=243.0 \mathrm{~nm})$ and a narrower $(\Delta \nu=5 \mathrm{GHz}$, ) infrared (IR) laser $\left(E_{\text {pulse }} \approx 8 \mathrm{~mJ}, \lambda=728-760 \mathrm{~nm}\right)$. The UV and IR laser light was linearly polarized, perpendicular and parallel to the magnetic field direction, respectively. Owing to the large Ps velocities, the limiting factor in the excitation efficiency was the $\sim 10 \%$ overlap of the UV laser bandwidth with the Doppler broadened $1^{3} S \rightarrow 2^{3} P$ transition; we estimate that $\sim 10^{3}$ Rydberg atoms were generated per second.

The Ps excitation region and the quadrupole guide structure and electric fields are shown schematically in Fig. 1. Because Ps is produced in a reflection geometry, the guide potentials are switched on $\sim 150 \mathrm{~ns}$ after the positron beam has passed through. Ps atoms are emitted within $10 \mathrm{~ns}$ of the positron implantation [52]; some of these atoms are excited to Rydberg levels and then travel towards the guide, reaching the entrance in $200 \mathrm{~ns}$. As indicated in Fig. 1(b), the path from the silica target to the guide is partially collimated by a $5.8 \mathrm{~mm}$ diameter hole in a ring electrode, located $6.0 \mathrm{~mm}$ from the target. Also shown in the figure are the positions of three lutetium-yttrium oxyorthosilicate (LYSO) scintillator-based gamma-ray detectors [55]. The first detector $(A)$ covers the target area and is used to monitor Ps formation and excitation, while Ps atoms that leave the excitation region are detected by two more detectors $(B$ and $C)$ located approximately $0.4 \mathrm{~m}$ away.
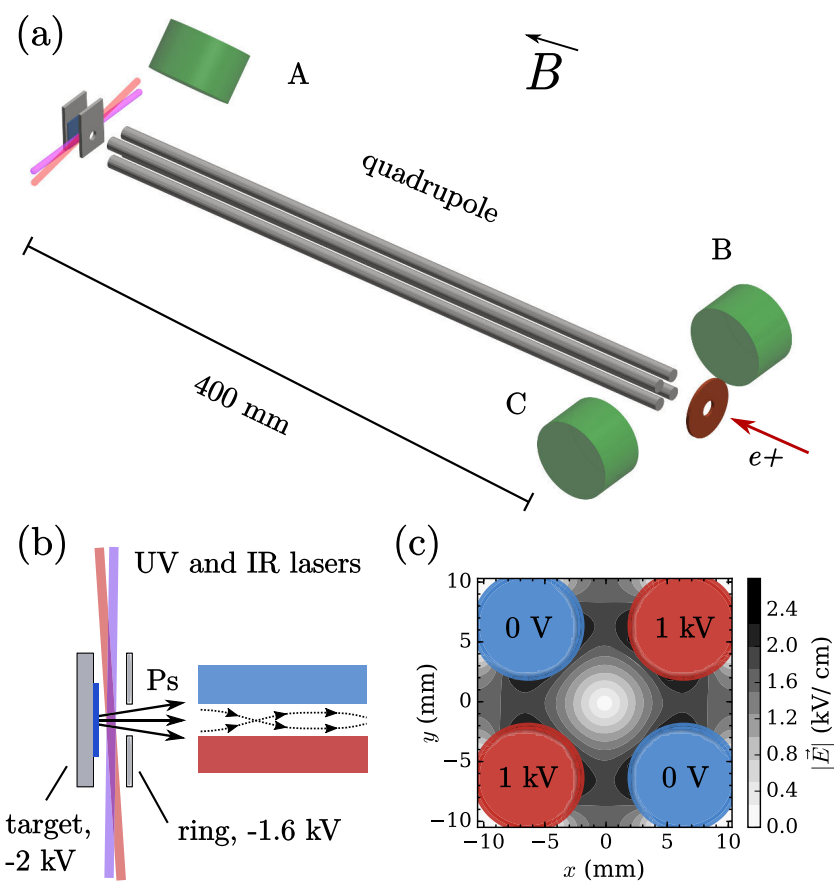

FIG. 1. (a) Schematic layout of the experiment, indicating the Ps excitation region, the position of the quadrupole guide, and the LYSO gamma-ray detectors $A, B$, and $C$. (b) Expanded view of the excitation region, and (c) a contour plot of the electric field strength inside the quadrupole with $1 \mathrm{kV}$ applied.

An electric field of $670 \mathrm{~V} \mathrm{~cm}^{-1}$ was applied in the excitation region in order to separate the Rydberg-Stark states, and the resulting Stark-broadened spectrum of transitions from $n=2$ to $n=10$ is shown in Fig. 2(a). These data were obtained (using detector $A$ ) by analyzing suitable time windows in single-shot lifetime spectra [56] to generate a signal parameter $S_{\gamma}$ that is proportional to the number of Rydberg atoms present [50]. There is a slight difference in the detection efficiency of lfs and hfs states because they are deflected by the inhomogeneous electric fields between the ring and guide electrodes. As a result, the measured spectrum is not perfectly symmetric. The applied field is not sufficient to permit the spectral resolution of individual Stark states [21], but it is sufficient to allow for the optical selection of predominantly lfs or hfs states. The $100 \mathrm{G}$ magnetic field used to transport the positrons does not significantly affect the energy level structure or dynamics of the Rydberg atoms. This is because in these states spin-orbit interactions are minimal, and the magnetic moment associated with the net orbital angular momentum of the electron-positron pair is zero [57].

The electric dipole moments of Ps Stark states are given by $\vec{\mu}=-(3 / 2) n k e a_{\mathrm{Ps}}$, where $k=n_{1}-n_{2}$ is the difference between the parabolic quantum numbers [25], $e$ is the electron charge, and $a_{\mathrm{Ps}}=2 a_{0}=1.058 \times 10^{-10} \mathrm{~m}$ is the Ps Bohr radius. For the laser polarizations employed here the excited states are produced predominantly with azimuthal quantum number $m_{\ell}=1$ [21], and even values of $k$, 

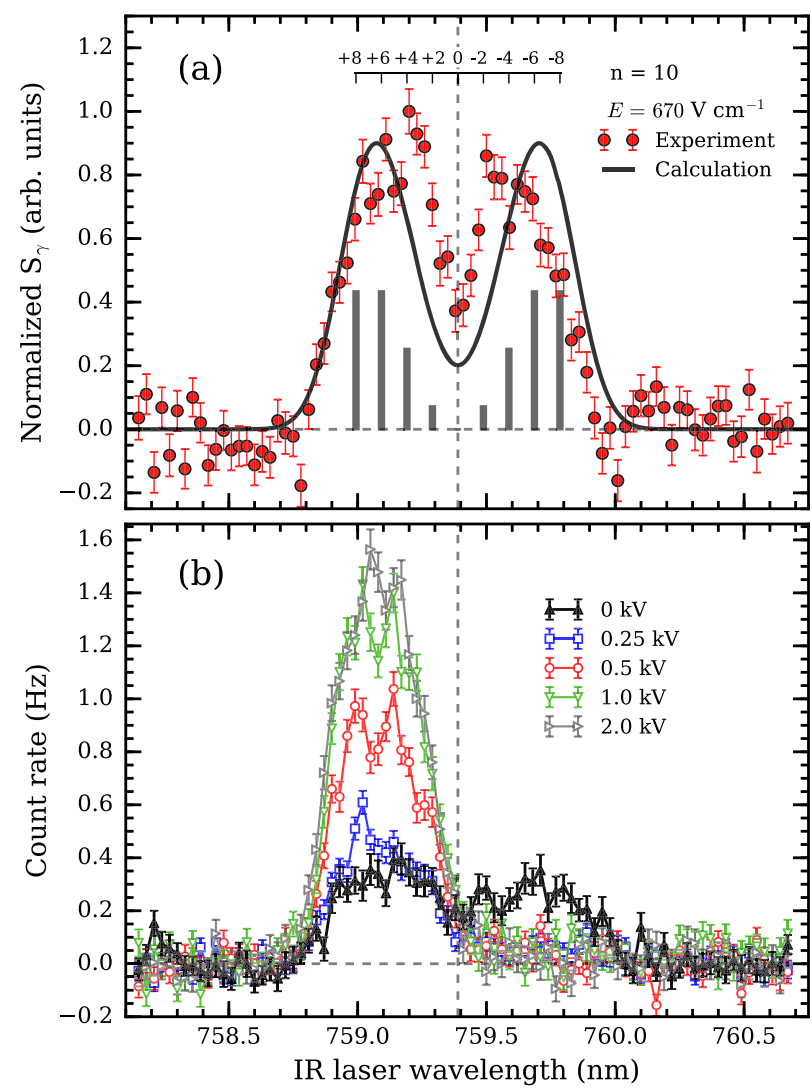

FIG. 2. (a) Spectrum of the $n=2 \rightarrow 10$ transitions measured using detector $A$. The grey vertical bars represent the relative spectral intensity for individual $k$ states, labeled by the horizontal scale. The black solid line is a convolution of these with the $\sim 130 \mathrm{GHz}$ experimental spectral resolution. (b) The background subtracted count rate obtained from detectors $B$ and $C$, measured with the indicated potentials applied to the guide. The dashed vertical line in both panels represents the expected centroid wavelength.

where $k$ has values from $-\left(n-\left|m_{\ell}\right|-1\right)$ to $+\left(n-\left|m_{\ell}\right|-1\right)$ in steps of 2 [25]. The calculated relative spectral intensities [58] of the transitions to the $n D$ components of the $m_{\ell}=1$ Stark states are indicated by the vertical bars in Fig. 2(a). The solid black line is a convolution of the calculated intensity distribution with a $130 \mathrm{GHz}$ Gaussian function, representing the experimental spectral resolution due to the laser bandwidth and Doppler broadening, as determined by field-free line shape measurements.

Enhanced Ps transport along the guide is indicated by an increased count rate in detectors $B$ and $C$. Unlike the single-shot lifetime spectra used to observe multiple nearsimultaneous events close to the Ps formation region, single event counting is used to detect atoms that travel away from this region. The counting procedure is the same as that used in previous Ps time-of-flight (TOF) measurements [22]. The detector signals are recorded with an oscilloscope in $6 \mu$ s time windows in which an event is registered if the output amplitude exceeds $1 \mathrm{mV}$ for more than $30 \mathrm{~ns}$. The overall efficiency of the two detectors, including the solid angles subtended, is estimated to be on the order of $5 \%$.

Figure 2(b) shows the background subtracted total count rate from detectors $B$ and $C$ as a function of the IR laser wavelength, for a range of voltages applied to the guide. The background was measured with the IR laser off resonance. The data obtained with $0 \mathrm{~V}$ applied to the guide were recorded at the same time as the spectrum shown in Fig. 2(a). In this case we detect any Ps Stark states that are emitted towards the guide within a narrow cone with a half angle of approximately $1^{\circ}$.

When the guide fields are applied the signal detected by $B$ and $C$ depends strongly on the IR laser wavelength. No hfs states are detected, demonstrating that even for states with the smallest electric dipole moments (i.e., $k=-2$ states with $\mu=150 \mathrm{D})$ and for the lowest applied fields, the hfs states cannot traverse the guide. The maximum applied potential results in a maximum electric field of $4.2 \mathrm{kV} \mathrm{cm}^{-1}$, which is well below the lowest field required to ionize Ps states with $n=10\left(\sim 14 \mathrm{kV} \mathrm{cm}^{-1}\right.$ [58]).

For a low guide field only those states with the highest dipole moment (i.e., $k=+8$ states with $\mu=610 \mathrm{D}$ ) are observed above the guide off background signal. Nevertheless, this field is sufficient to suppress transport of all hfs states. When the field is increased, more of the lfs Stark states are transported, which may be partially due to a focusing effect in the space between the ring and guide electrodes.

The TOF distributions of the guided atoms have been directly measured for two different IR wavelengths, for which either lfs $(k \sim+6)$ or hfs $(k \sim-6)$ Stark states were predominantly prepared. These data are shown in Fig. 3.

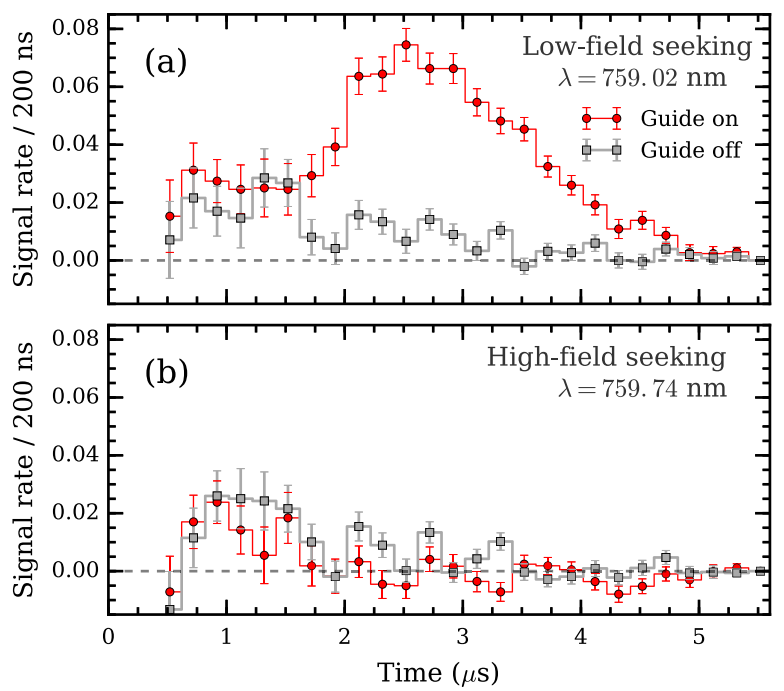

FIG. 3. Background subtracted TOF data recorded by detectors $B$ and $C$ with $1 \mathrm{kV}$ applied to the guide electrodes, for IR laser wavelengths corresponding to (a) outer lfs $(k \sim+6)$ and (b) outer hfs $(k \sim-6)$ states, as indicated. The data set is truncated for times less than $\sim 0.5 \mu$ s because of detector saturation following implantation of the positron pulse. 


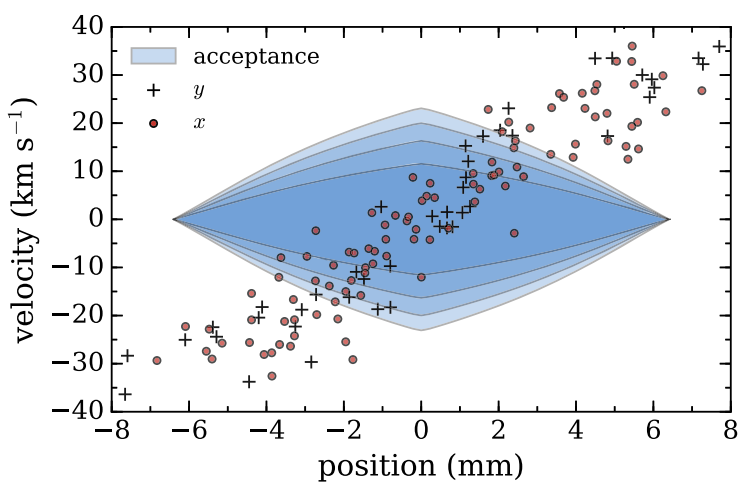

FIG. 4. Transverse phase-space acceptance of the guide for $n=10$ Ps atoms with $+1 \mathrm{kV}$ applied to the electrodes. The concentric diamonds indicate the acceptance for Stark states with $k=+2,+4,+6$, and +8 . The symbols indicate the initial positions in phase space in the $x$ and $y$ directions of 100 Rydberg atoms, as described in the text.

The measured TOF distributions are consistent with the fraction of the initial Ps phase-space distribution that meets the guide acceptance criteria. The flight times are comparable to the $3 \mu \mathrm{s}$ florescence lifetime of Rydberg-Stark states with $n=10$ [22]. This process results in selfannihilation in the field of view of detectors $B$ and $C$, while atoms that do not decay via fluorescence mostly annihilate following a collision with a pumping restriction at the end of the quadrupole structure (see Fig. 1).

Whether a Ps atom is guided or not depends on its radial position and velocity at the guide entrance, its dipole moment, and the electric fields in the quadrupole structure. The transverse phase-space acceptance when the guide is operated at $+1 \mathrm{kV}$ is shown in Fig. 4 for each lfs Stark state. Also shown are the initial positions in phase space in the $x$ and $y$ directions of 100 Rydberg atoms. The trajectories have been determined numerically, assuming a $700 \mathrm{~K}$ Maxwell-Boltzmann velocity distribution and a cosine angular distribution. Note that the velocity spread in the $x$ direction is narrowed by the Doppler selection of the excitation lasers. The trajectories, neglecting any focusing effects, indicate that $\sim 10 \%$ of the Rydberg Ps atoms that pass through the ring electrode are guided. This corresponds to $\sim 8 \%$ of the total Rydberg Ps distribution.

The design of the guide used in this work is very basic, and can be improved in numerous ways. For example, better matching of the excitation region and the guide entrance will increase the geometrical acceptance. Introducing multiple guide stages will make it possible to perform deceleration experiments, and sets the stage for further work using time-varying fields. It may also be beneficial to use different electrode and electric field configurations, such as surface based transmission lines $[59,60]$ or wires $[61,62]$, which would be more convenient for probing guided Ps atoms with microwave or laser fields, or for allowing them to interact with other species (e.g., $[63,64])$.

The $\sim 25 \mathrm{meV}$ kinetic energy of thermal Ps is well within the range of energy extraction achieved in other Rydberg-Stark deceleration and trapping experiments using time-varying fields [60]. Therefore, with some modifications to account for the different velocity distributions, similar levels of control are anticipated in Ps experiments. Since Stark deceleration operates via conservative forces, it cannot be exploited to reduce the phase-space density of (i.e., to cool) a sample of atoms or molecules. The efficacy of such techniques, as well as those employing velocity selection (for example, as might be achieved using a curved quadrupole guide [65]), improves when colder sources of atoms or molecules are employed. Thus, the development of methods to generate cold or monoenergetic Ps atoms (e.g., [20]) will be beneficial for deceleration and trapping experiments.

The experiments described here represent an important step towards the long-term goal of using inhomogeneous electric fields to create controlled beams of Ps atoms in long-lived Rydberg-Stark states. The ability to simultaneously extend the lifetime and control the translational motion of Ps atoms will facilitate several ongoing research programs, such as spectroscopy [19,66], gravitational free-fall experiments $[67,68]$, generating positron-atom bound states via Ps-atom scattering [63], and the formation of antihydrogen following Rydberg Ps interactions with antiprotons [64].

This work was supported by UCL through its Impact Studentship Programme, and was funded in part by the ERC (Grant No. CIG 630119) and the EPSRC (Grant No. EP/K028774/1). We are grateful to L. Liszkay for providing silica samples.

[1] C. E. Wieman, D. E. Pritchard, and D. J. Wineland, Atom cooling, trapping, and quantum manipulation, Rev. Mod. Phys. 71, S253 (1999).

[2] S. Y. T. van de Meerakker, H. L. Bethlem, N. Vanhaecke, and G. Meijer, Manipulation and control of molecular beams, Chem. Rev. 112, 4828 (2012).

[3] H. L. Bethlem, G. Berden, and G. Meijer, Decelerating Neutral Dipolar Molecules, Phys. Rev. Lett. 83, 1558 (1999).

[4] S. D. Hogan, Rydberg-Stark deceleration of atoms and molecules, EPJ Techniques and Instrumentation 3, 1 (2016).

[5] R. Fulton, A. I. Bishop, and P. F. Barker, Optical Stark Decelerator for Molecules, Phys. Rev. Lett. 93, 243004 (2004).

[6] N. Vanhaecke, U. Meier, M. Andrist, B. H. Meier, and F. Merkt, Multistage Zeeman deceleration of hydrogen atoms, Phys. Rev. A 75, 031402 (2007).

[7] I. Bloch, J. Dalibard, and W. Zwerger, Many-body physics with ultracold gases, Rev. Mod. Phys. 80, 885 (2008). 
[8] J. Baron, W. C. Campbell, D. DeMille, J. M. Doyle, G. Gabrielse, Y. V. Gurevich, P. W. Hess, N. R. Hutzler, E. Kirilov, I. Kozyryev, B. R. O’Leary, C. D. Panda, M. F. Parsons, E. S. Petrik, B. Spaun, A. C. Vutha, and A. D. West, Order of magnitude smaller limit on the electric dipole moment of the electron, Science 343, 269 (2014).

[9] A. D. Ludlow, M. M. Boyd, J. Ye, E. Peik, and P. O. Schmidt, Optical atomic clocks, Rev. Mod. Phys. 87, 637 (2015).

[10] M. T. Bell and T. P. Softley, Ultracold molecules and ultracold chemistry, Mol. Phys. 107, 99 (2009).

[11] A. D. Cronin, J. Schmiedmayer, and D. E. Pritchard, Optics and interferometry with atoms and molecules, Rev. Mod. Phys. 81, 1051 (2009).

[12] A. Rich, Recent experimental advances in positronium research, Rev. Mod. Phys. 53, 127 (1981).

[13] P. G. Coleman, Positron Beams and their Applications, edition 1st ed. (World Scientific Publishing Co., Singapore, 2000).

[14] A. Ore and J. L. Powell, Three-photon annihilation of an electron-positron pair, Phys. Rev. 75, 1696 (1949).

[15] J. R. Danielson, D. H. E. Dubin, R. G. Greaves, and C. M. Surko, Plasma and trap-based techniques for science with positrons, Rev. Mod. Phys. 87, 247 (2015).

[16] D. B. Cassidy, S. H. M. Deng, R. G. Greaves, and A. P. Mills, Jr., Accumulator for the production of intense positron pulses, Rev. Sci. Instrum. 77, 073106 (2006).

[17] S. Chu and A. P. Mills, Jr., Excitation of the Positronium $1^{3} S_{1} \rightarrow 2^{3} S_{1}$ Two-Photon Transition, Phys. Rev. Lett. 48, 1333 (1982).

[18] D. B. Cassidy, T. H. Hisakado, H. W. K. Tom, and A. P. Mills, Jr., Efficient Production of Rydberg Positronium, Phys. Rev. Lett. 108, 043401 (2012).

[19] A. C. L. Jones, T. H. Hisakado, H. J. Goldman, H. W. K. Tom, A. P. Mills, Jr., and D. B. Cassidy, Doppler-corrected Balmer spectroscopy of Rydberg positronium, Phys. Rev. A 90, 012503 (2014).

[20] A. C. L. Jones, H. J. Goldman, Q. Zhai, P. Feng, H. W. K. Tom, and A.P. Mills, Jr., Monoenergetic Positronium Emission from Metal-Organic Framework Crystals, Phys. Rev. Lett. 114, 153201 (2015).

[21] T. E. Wall, A. M. Alonso, B.S. Cooper, A. Deller, S. D. Hogan, and D. B. Cassidy, Selective Production of RydbergStark States of Positronium, Phys. Rev. Lett. 114, 173001 (2015).

[22] A. Deller, A. M. Alonso, B. S. Cooper, S. D. Hogan, and D. B. Cassidy, Measurement of Rydberg positronium fluorescence lifetimes, Phys. Rev. A 93, 062513 (2016).

[23] A. I. Alekseev, Two-photon annihilation of positronium in the $P$ state, Sov. Phys. JETP 7, 826 (1958).

[24] A. I. Alekseev, Three-photon annihilation of positronium in the $P$ state, Sov. Phys. JETP 9, 1312 (1959).

[25] T.F. Gallagher, Rydberg Atoms (publisher Cambridge University Press, Cambridge, 1994).

[26] W. H. Wing, Electrostatic Trapping of Neutral Atomic Particles, Phys. Rev. Lett. 45, 631 (1980).

[27] T. Breeden and H. Metcalf, Stark Acceleration of Rydberg Atoms in Inhomogeneous Electric Fields, Phys. Rev. Lett. 47, 1726 (1981).

[28] H. G. Bennewitz, W. Paul, and Ch. Schlier, Fokussierung polarer moleküle, Z. Phys. 141, 6 (1955).
[29] R. Paulin and G. Ambrosino, Annihilation libre de l'orthopositonium formé dans certaines poudres de grande surface spécifique, J. Phys. II (France) 29, 263 (1968).

[30] A. P. Mills, Jr. and Loren Pfeiffer, Desorption of Surface Positrons: A Source of Free Positronium at Thermal Velocities, Phys. Rev. Lett. 43, 1961 (1979).

[31] P. Sferlazzo, S. Berko, K. G. Lynn, A. P. Mills, Jr., L. O. Roellig, A. J. Viescas, and R.N. West, Evidence for Phonon-Assisted Positronium Emission from Graphite, Phys. Rev. Lett. 60, 538 (1988).

[32] L. Liszkay, C. Corbel, P. Perez, P. Desgardin, M. F. Barthe, T. Ohdaira, R. Suzuki, P. Crivelli, U. Gendotti, A. Rubbia, M. Etienne, and A. Walcarius, Positronium reemission yield from mesostructured silica films, Appl. Phys. Lett. 92, 063114 (2008).

[33] D. B. Cassidy, T. H. Hisakado, H. W. K. Tom, and A. P. Mills, Jr., New Mechanism for Positronium Formation on a Silicon Surface, Phys. Rev. Lett. 106, 133401 (2011).

[34] P. Crivelli, D. Cooke, B. Barbiellini, B. L. Brown, J. I. Feldblyum, P. Guo, D. W. Gidley, L. Gerchow, and A. J. Matzger, Positronium emission spectra from self-assembled metal-organic frameworks, Phys. Rev. B 89, 241103 (2014).

[35] G. Scholes, Atomic and Molecular Beam Methods (Oxford University Press, New York, 1988), Vol. 1.

[36] S. E. Maxwell, N. Brahms, R. deCarvalho, D. R. Glenn, J. S. Helton, S. Nguyen, D. Patterson, J. M. Doyle, D. R. Glenn, J. Petricka, and D. DeMille, High-Flux Beam Source for Cold, Slow Atoms or Molecules, Phys. Rev. Lett. 95, 173201 (2005).

[37] A. P. Mills, Jr., E. D. Shaw, R. J. Chichester, and D. M. Zuckerman, Positronium thermalization in $\mathrm{SiO}_{2}$ powder, Phys. Rev. B 40, 2045 (1989).

[38] A. P. Mills, Jr., E. D. Shaw, M. Leventhal, R. J. Chichester, and D. M. Zuckerman, Thermal desorption of cold positronium from oxygen-treated $\mathrm{Al}(111)$ surfaces, Phys. Rev. B 44, 5791 (1991).

[39] S. Mariazzi, P. Bettotti, and R. S. Brusa, Positronium Cooling and Emission in Vacuum from Nanochannels at Cryogenic Temperature, Phys. Rev. Lett. 104, 243401 (2010).

[40] L. Liszkay, F. Guillemot, C. Corbel, J.-P. Boilot, T. Gacoin, E. Barthel, P. Perez, M.-F. Barthe, P. Desgardin, P. Crivelli, U. Gendotti, and A. Rubbia, Positron annihilation in latextemplated macroporous silica films: pore size and orthopositronium escape, New J. Phys. 14, 065009 (2012).

[41] B. S. Cooper, A. M. Alonso, A. Deller, L. Liszkay, and D. B. Cassidy, Positronium production in cryogenic environments, Phys. Rev. B 93, 125305 (2016).

[42] E. P. Liang and C. D. Dermer, Laser cooling of positronium, Opt. Commun. 65, 419 (1988).

[43] D. B. Cassidy, H. W. K. Tom, and A. P. Mills, Jr., Fundamental physics with cold positronium, AIP Conf. Proc. 1037, 66 (2008).

[44] H. Iijima, T. Asonuma, T. Hirose, M. Irako, T. Kumita, M. Kajita, K. Matsuzawa, and K. Wada, Laser cooling system of ortho-positronium, Nucl. Instrum. Methods Phys. Res., Sect. A 455, 104 (2000).

[45] D. B. Cassidy, P. Crivelli, T. H. Hisakado, L. Liszkay, V. E. Meligne, P. Perez, H. W. K. Tom, and A. P. Mills, Jr., Positronium cooling in porous silica measured via Doppler spectroscopy, Phys. Rev. A 81, 012715 (2010). 
[46] D. Townsend, A. L. Goodgame, S. R. Procter, S. R. Mackenzie, and T. P. Softley, Deflection of krypton Rydberg atoms in the field of an electric dipole, J. Phys. B 34, 439 (2001).

[47] Y. Yamakita, S. R. Procter, A. L. Goodgame, T. P. Softley, and F. Merkt, Deflection and deceleration of hydrogen Rydberg molecules in inhomogeneous electric fields, J. Chem. Phys. 121, 1419 (2004).

[48] E. Vliegen and F. Merkt, On the electrostatic deceleration of argon atoms in high Rydberg states by time-dependent inhomogeneous electric fields, J. Phys. B 38, 1623 (2005).

[49] S. D. Hogan and F. Merkt, Demonstration of ThreeDimensional Electrostatic Trapping of State-Selected Rydberg Atoms, Phys. Rev. Lett. 100, 043001 (2008).

[50] B. S. Cooper, A. M. Alonso, A. Deller, T. E. Wall, and D. B. Cassidy, A trap-based pulsed positron beam optimized for positronium laser spectroscopy, Rev. Sci. Instrum. 86, 103101 (2015).

[51] A.P. Mills, Jr., Time bunching of slow positrons for annihilation lifetime and pulsed laser photon absorption experiments, J. Appl. Phys. 22, 273 (1980).

[52] A. Deller, B. S. Cooper, T. E. Wall, and D. B. Cassidy, Positronium emission from mesoporous silica studied by laser-enhanced time-of-flight spectroscopy, New J. Phys. 17, 043059 (2015).

[53] P. Crivelli, U. Gendotti, A. Rubbia, L. Liszkay, P. Perez, and C. Corbel, Measurement of the orthopositronium confinement energy in mesoporous thin films, Phys. Rev. A 81, 052703 (2010).

[54] K. P. Ziock, R. H. Howell, F. Magnotta, R. A. Failor, and K. M. Jones, First Observation of Resonant Excitation of High$n$ States in Positronium, Phys. Rev. Lett. 64, 2366 (1990).

[55] A. M. Alonso, B. S. Cooper, A. Deller, and D. B. Cassidy, Single-shot positron annihilation lifetime spectroscopy with LYSO scintillators, Nucl. Instrum. Methods Phys. Res., Sect. A 828, 163 (2016).

[56] D. B. Cassidy, S. H. M. Deng, H. K. M. Tanaka, and A. P. Mills, Jr., Single-shot positron annihilation lifetime spectroscopy, Appl. Phys. Lett. 88, 194105 (2006).
[57] S. M. Curry, Combined Zeeman and motional Stark effects in the first excited state of positronium, Phys. Rev. A 7, 447 (1973).

[58] S. D. Hogan, Calculated photoexcitation spectra of positronium Rydberg states, Phys. Rev. A 87, 063423 (2013).

[59] Yong Xia, Yaling Yin, Haibo Chen, Lianzhong Deng, and Jianping Yin, Electrostatic Surface Guiding for Cold Polar Molecules: Experimental Demonstration, Phys. Rev. Lett. 100, 043003 (2008).

[60] P. Lancuba and S. D. Hogan, Electrostatic trapping and in situ detection of Rydberg atoms above chip-based transmission lines, J. Phys. B 49, 074006 (2016).

[61] H. J. Loesch and B. Scheel, Molecules on Kepler Orbits: An Experimental Study, Phys. Rev. Lett. 85, 2709 (2000).

[62] H. Ko and S. D. Hogan, High-field-seeking Rydberg atoms orbiting a charged wire, Phys. Rev. A 89, 053410 (2014).

[63] A. R. Swann, D. B. Cassidy, A. Deller, and G. F. Gribakin, Formation of positron-atom bound states in collisions between Rydberg Ps and neutral atoms, Phys. Rev. A 93, 052712 (2016).

[64] A. S. Kadyrov, C. M. Rawlins, A. T. Stelbovics, I. Bray, and M. Charlton, Antihydrogen Formation via Antiproton Scattering with Excited Positronium, Phys. Rev. Lett. 114, 183201 (2015).

[65] S. A. Rangwala, T. Junglen, T. Rieger, P. W. H. Pinkse, and G. Rempe, Continuous source of translationally cold dipolar molecules, Phys. Rev. A 67, 043406 (2003).

[66] S. G. Karshenboim, Precision study of positronium: testing bound-state QED theory, Int. J. Mod. Phys. A A19, 3879 (2004).

[67] A. P. Mills, Jr. and M. Leventhal, Can we measure the gravitational free fall of cold Rydberg state positronium?, Nucl. Instrum. Methods Phys. Res., Sect. B 192, 102 (2002).

[68] D. B. Cassidy and S. D. Hogan, Atom control and gravity measurements using Rydberg positronium, Int. J. Mod. Phys. Conf. Ser. 30, 1460259 (2014). 